\title{
Sodium-glucose cotransporter-2 inhibitors (SGLT-2i) in the perioperative setting
}

\author{
Sharon C. Peacock, BSc(PT), MD, FRCPC (1D · Julie A. Lovshin, MD, PhD
}

Received: 20 October 2017/ Accepted: 23 October 2017/Published online: 20 November 2017

(c) Canadian Anesthesiologists' Society 2017

Sodium-glucose cotransporter-2 inhibitors (SGLT-2i) are the newest class of oral antihyperglycemic agents approved for treating type 2 diabetes mellitus (T2DM). Mechanistically, SGLT-2i reduce blood glucose by promoting glucosuria through inhibition of sodiumglucose co-transporter-2 (SGLT-2) channels in the epithelium of the renal proximal tubules. SGLT-2i modestly reduce blood glucose concentrations $(\mathrm{HbAl} 1 \mathrm{c} \sim$ -0.7 to $-1.0 \%),{ }^{1}$ with glucoregulatory effects that occur independent of insulin secretion; hence, they are associated with a low risk of hypoglycemia relative to insulin secretory agents. ${ }^{2}$ In light of their favourable benefit-torisk profile, ease of use (once daily oral administration), and ancillary benefits on total body weight loss $(\sim 3 \mathrm{~kg})$ and blood pressure (BP) reduction (systolic BP -4 to -6 $\mathrm{mmHg}$, diastolic BP -1 to $-2 \mathrm{mmHg}$ ), SGLT-2i are now routinely used in practice. Some agents in this drug class are now prioritized (e.g., empagliflozin) as preferred second line therapy in patients with clinical cardiovascular disease, after metformin. ${ }^{1,3}$ These recommendations followed the Empagliflozin Cardiovascular Outcome Event Trial in Type 2 Diabetes Mellitus Patients (EMPA-REG OUTCOME) trial, a cardiovascular safety study in (T2DM) patients with established clinical cardiovascular disease. ${ }^{4}$ Empagliflozin

S. C. Peacock, BSc(PT), MD, FRCPC ( $\square)$

Department of Anesthesiology, University of Toronto, Room 1200, 12th Floor, 123 Edward Street, Toronto, ON M5G 1E2, Canada

e-mail: sharon.c.peacock@gmail.com

J. A. Lovshin, MD, PhD

Division of Endocrinology and Metabolism, Department of Medicine, Sunnybrook Health Sciences Centre, University of Toronto, Toronto, ON, Canada significantly reduced the risk of cardiovascular mortality (38\%), hospitalization for heart failure $(35 \%),{ }^{4}$ and nephropathy events (39\%). ${ }^{5}$ The combined results of two more recent cardiovascular safety studies with the SGLT2i, canagliflozin (CANagliflozin cardioVascular Assessment Study [CANVAS] and CANagliflozin cardioVascular Assessment Study-Renal [CANVAS-R]), known collectively as the CANVAS program, were also consistent with cardiac and renal protection in a large T2DM population at high cardiovascular risk. ${ }^{6}$ In light of these clinical trial data, SGLT-2i will likely be encountered more frequently by anesthesiologists in the perioperative period.

Amidst growing enthusiasm for SGLT-2i, anesthesiologists should be aware of their associated risk profile, particularly the very rare $(0.1 \%)$ but serious risk for diabetic ketoacidosis (DKA). ${ }^{7}$ Although incompletely understood, DKA risk with SGLT-2i maybe increased because of several precipitating factors, including fasting, dehydration, and surgical stress. ${ }^{8}$ Furthermore, diagnosis of DKA, particularly euglycemic DKA (euDKA), might be delayed because of overlap between the clinical signs and symptoms of DKA with non-specific ones arising perioperatively (Figure).

Unfortunately, consensus guidance around the perioperative use of SGLT-2i has yet to emerge. In this issue of the Journal, Lau et al. present three cases of euDKA occurring on postoperative day 1 after elective coronary artery bypass grafting following the discontinuation of empagliflozin $24-48 \mathrm{hr}$ preoperatively. ${ }^{9}$ Their case series demonstrates that the optimal preoperative regimen for discontinuation of SGLT-2i has yet to be established and that heightened awareness of SGLT-2i-assoicated DKA risk should promote earlier recognition and management. Within their hospital-based 


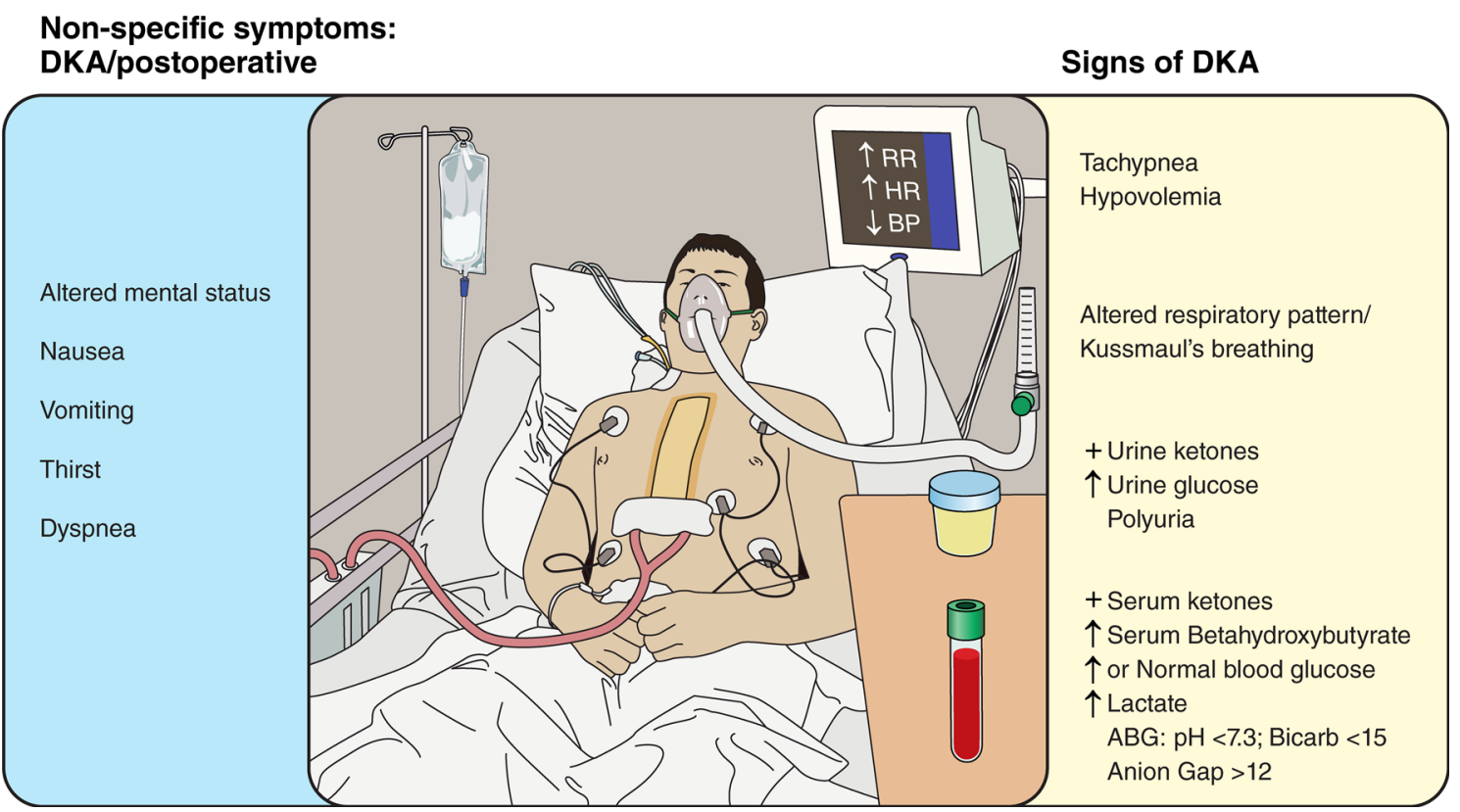

Figure Non-specific postoperative signs and symptoms overlapping with diabetic ketoacidosis. $\mathrm{ABG}=$ arterial blood gas; Bicarb $=$ bicarbonate; DKA = diabetic ketoacidosis

practice, these cases elicited changes to several perioperative management procedures including planning around glucose control/insulin administration, preoperative discontinuation/postoperative resumption of SGLT-2i, and vigilance on volume optimization and analgesia to minimize counter-regulatory hormone stimulation. The cases highlight the need for education and consensus guidance on perioperative use of SGLT-2i, particularly in high-risk surgical settings such as cardiopulmonary bypass, wherein DKA risk may be elevated.

Mechanistically, how SGLT-2i contribute to an increased risk for DKA is not completely understood. Sodium-glucose cotransporter-2 inhibitors increase glucagon secretion due to direct effects on alpha cells expressing SGLT-2 channels, resulting in hyperglucagonemia, stimulating hepatic glucose production, leading to lipolysis and increasing ketone production. ${ }^{10}$ Sodium-glucose cotransporter-2 inhibition is also associated with a shift from carbohydrate to lipid metabolism, further promoting lipolysis, ketogenesis, and increased $\beta$-hydroxybutyrate production (ketogenic substrates that are already increased during times of stress). ${ }^{11}$ Additionally, as a consequence of decreased proximal tubular sodium reabsorption, renal ketone reabsorption is increased. ${ }^{2}$ Furthermore, due to the glucuretic effects of these drugs, blood glucose concentrations may be near normal or only slightly elevated, which may hinder early detection of DKA. From a hemodynamic perspective, the natriuretic effects of
Signes et symptômes postopératoires non spécifiques chevauchant ceux de l'acidocétose diabétique. GSA = gazométrie du sang artériel; Bicarb = bicarbonate; $\mathrm{ACD}=$ acidocétose diabétique

SGLT-2i promote plasma volume reduction and reduce BP and arterial stiffness. ${ }^{2}$ If not optimized preoperatively, volume depletion could intensify the risk of hemodynamic effects associated with DKA. Major surgery has been implicated as a precipitating factor for euDKA, as supplemental insulin needs can be underestimated postoperatively because of a reduction in endogenous insulin secretion and utilization from the stress response of undergoing surgery. ${ }^{8}$ Moreover, the insulin requirements necessary to suppress ketosis/metabolic acidosis may be underestimated because of near normal blood glucose concentrations associated with SGLT-2i use. ${ }^{8}$

The debate, however, continues over whether SGLT-2i are actually causative for DKA in the setting of T2DM. ${ }^{12}$ In a meta-analysis by Tang et al., in a total of ten eligible RCTs involving 13,134 patients, only 14 DKA events in T2DM patients associated with SGLT-2i use were identified. Overall, the event rates were $0.1 \%$ in the group of SGLT-2i users $v s \quad 0.06 \%$ in the other glucoselowering groups (odds ratio [OR], 1.71; 95\% confidence interval [CI], 0.56 to 5.2), with subgroup analyses showing that SGLT-2i were not significantly associated with an increased risk of DKA when compared with placebo (OR, $1.98 ; 95 \%$ CI, 0.56 to 6.9 ). ${ }^{7}$ A meta-analysis by Monami et al. included randomized trials with a treatment duration $>12$ weeks, patients with T2DM, and comparing a SGLT$2 \mathrm{i}$ with placebo or active comparators. Out of 72 trials, 9 reported at least one event of ketoacidosis, with 10,157 and 5,396 subjects in the SGLT-2i and comparator groups 
respectively. The number of events of DKA was 16 for SGLT-2i and 6 for comparators. No signal of increased risk for ketoacidosis was observed for SGLT-2i as a class (OR, $1.14 ; 95 \% \mathrm{CI}, 0.45$ to $2.9 ; P=0.78$ ). The authors concluded that the risk of DKA associated with SGLT-2i is negligible when properly prescribed in patients with T2DM. ${ }^{13}$ Analyses of the Federal Drug Agency's Adverse Event Reporting System by Fadini reviewed 2,397 case reports of DKA associated with SGLT-2i use from 2014 to 2016. The authors concluded that increased reporting of DKA, associated with SGLT-2i use, was likely attributed to increased pharmacovigilance and reporting bias rather than indicative of true incidence, which may be better inferred from real-world studies and large clinical trials. ${ }^{14}$

While it seems reasonable to propose recommendations around perioperative use of SGLT-2i based on pharmacokinetics as proposed by Lau et al., there is a noteworthy lack of consensus recommendations from major diabetes, surgical, or anesthesia associations for perioperative use of SGLT-2i, which we recently highlighted elsewhere. ${ }^{15}$ Although the American Association of Clinical Endocrinologists and the American College of Endocrinology recommend discontinuation of SGLT-2i $24 \mathrm{hr}$ preoperatively, ${ }^{16}$ the case series by Lau et al. (and others) ${ }^{8,12}$ emphasizes that euDKA can still precipitate despite $48 \mathrm{hr}$ perioperative discontinuation of SGLT-2i. ${ }^{12}$ This highlights an important clinical knowledge gap around SGLT-2i use in the perioperative setting and represents an opportunity for quality improvement research.

\section{Les inhibiteurs du cotransporteur sodium- glucose de type 2 (SGLT-2i) dans le contexte périopératoire}

Les inhibiteurs du cotransporteur sodium-glucose de type 2 (SGLT-2i) sont la classe la plus récente d'agents antihyperglycémiques oraux approuvés pour le traitement du diabète de type 2 . D'un point de vue physiologique, les SGLT-2i réduisent la glycémie en promouvant une glycosurie par l'inhibition des canaux du cotransporteur sodium-glucose de type 2 (SGLT-2) dans l'épithélium des tubules proximaux rénaux. Les SGLT-2i réduisent de façon modeste les concentrations glycémiques $(\mathrm{HbA} 1 \mathrm{c} \sim-0,7$ à $-1,0 \%),{ }^{1}$ ayant des effets de glycorégulation survenant indépendamment de la sécrétion d'insuline; ainsi, ils sont associés à un faible risque d'hypoglycémie relative aux agents sécréteurs d'insuline. ${ }^{2}$ À la lumière de leur profil bénéfique avantageux par rapport aux risques qu'ils comportent, de leur facilité d'utilisation (une administration orale quotidienne), et des avantages connexes sur la perte de poids totale $(\sim 3 \mathrm{~kg})$ et la réduction de la tension artérielle (TA) (TA systolique -4 à $-6 \mathrm{mmHg}$, TA diastolique -1 à -2 mmHg), les SGLT-2i sont aujourd'hui couramment utilisés en clinique. Certains agents de cette classe de médicaments sont aujourd'hui priorisés (notamment l'empagliflozin) en tant que traitement de prédilection de deuxième ligne, après la metformine, chez les patients souffrant de maladie cardiovasculaire clinique. ${ }^{1,3}$ Ces recommandations font suite à l'étude EMPA-REG OUTCOME (Empagliflozin Cardiovascular Outcome Event Trial in Type 2 Diabetes Mellitus Patients), une étude d'innocuité cardiovasculaire réalisée auprès de patients diabétiques de type 2 souffrant de maladie cardiovasculaire clinique établie. ${ }^{4}$ L'empagliflozin a réduit de manière significative le risque de mortalité cardiovasculaire (38\%), l'hospitalisation pour insuffisance cardiaque $(35 \%)^{4}$ et les complications liées à une néphropathie $(39 \%) .{ }^{5}$ Les résultats combinés de deux études d'innocuité cardiovasculaire plus récentes portant sur un SGLT-2i, le canagliflozin (l'étude CANVAS [CANagliflozin cardioVascular Assessment Study] et l'étude CANVAS-R [CANagliflozin cardioVascular Assessment Study-Renal]), connues collectivement en tant que programme CANVAS, ont également démontré une protection cardiaque et rénale chez une grande population de personnes diabétiques à risque cardiovasculaire élevé. ${ }^{6}$ À la lumière de ces données d'études cliniques, les anesthésiologistes vont probablement remarquer une présence plus fréquente des SGLT-2i en période périopératoire.

$\mathrm{Au}$ milieu de l'enthousiasme grandissant que rencontrent les SGLT-2i, les anesthésiologistes devraient garder à l'esprit le profil de risque qui leur est associé, et tout particulièrement le risque, certes très rare $(0,1 \%)$, mais grave, d'acidocétose diabétique. ${ }^{7}$ Bien qu'encore mal compris, le risque d'acidocétose diabétique pourrait être accru avec les SGLT-2i en raison de plusieurs facteurs précipitants, notamment le jeûne, la déshydratation et le stress chirurgical. ${ }^{8}$ En outre, le diagnostic d'acidocétose diabétique, particulièrement d'acidocétose diabétique euglycémique, pourrait être retardé en raison du chevauchement des signes et symptômes cliniques de l'acidocétose diabétique et des signes et symptômes non spécifiques survenant en période périopératoire (voir Figure).

À ce jour, il n'existe malheureusement pas encore de recommandations consensuelles concernant l'utilisation 
périopératoire des SGLT-2i. Dans ce numéro du Journal, Lau et coll. présentent trois cas d'acidocétose euglycémique survenus le premier jour postopératoire après une chirurgie de pontage aortocoronarien non urgente; ces cas ont été observés subséquemment à l'interruption d'un traitement d'empagliflozin 24-48 h avant l'opération. ${ }^{9}$ Leur série de cas démontre que le régime préopératoire optimal pour interrompre les SGLT2i n'a pas encore été établi et qu'une meilleure prise de conscience du risque d'acidocétose diabétique associé aux SGLT-2i devrait encourager son identification et sa prise en charge plus précoce. Dans le cadre de la pratique hospitalière des auteurs, ces cas ont motivé la modification de plusieurs processus de prise en charge périopératoire, notamment de la planification entourant le contrôle glycémique et l'administration d'insuline, de l'interruption pré- et de la reprise postopératoire de l'administration de SGLT-2i, et de la vigilance concernant l'optimisation volumique et l'analgésie afin de minimiser la stimulation hormonale contre-régulatrice. Ces cas soulignent le besoin de formation et de recommandations consensuelles quant à l'utilisation périopératoire des SGLT-2i, particulièrement dans des contextes chirurgicaux à risque élevé tels que la circulation extracorporelle, au cours de laquelle le risque d'acidocétose diabétique pourrait être plus élevé.

D'un point de vue physiologique, nous ne comprenons que partiellement la façon dont les SGLT-2i contribuent à accroître le risque d'acidocétose diabétique. Les SGLT-2i augmentent la sécrétion de glucagon en raison de leurs effets directs sur les cellules alpha exprimant les canaux des SGLT-2, ce qui provoque une hyperglucagonémie, stimule la production de glucose hépatique, résulte en une lipolyse et augmente la production de cétone. ${ }^{10}$ L'inhibition des SGLT-2i est également associée à un changement de métabolisme des hydrates de carbone/ glucides vers celui des lipides, ce qui favorise encore plus la lipolyse, la cétogenèse, et une augmentation de la production de $\beta$-hydroxybutyrate (des substrats cétogènes déjà accrus en période de stress). ${ }^{11}$ En outre, en conséquence d'une diminution de la réabsorption sodique dans les tubules proximaux, la réabsorption rénale de la cétone augmente. $^{2}$ De plus, en raison des effets glucorétiques de ces médicaments, les concentrations glycémiques pourraient s'approcher de la normale ou n'être que légèrement élevées, ce qui pourrait restreindre le dépistage précoce d'une acidocétose diabétique. Du point de vue de l'hémodynamie, les effets natriurétiques des SGLT-2i favorisent une réduction du volume plasmatique et réduisent la TA et la rigidité des parois artérielles. ${ }^{2} \mathrm{Si}$ la volémie n'a pas été optimisée en période préopératoire, une déplétion volumique pourrait intensifier le risque d'effets hémodynamiques associés à l'acidocétose diabétique. Les chirurgies majeures ont été mises en cause comme facteurs précipitants d'acidocétose diabétique euglycémique, étant donné que les besoins en insuline complémentaire pourraient être sous-estimés en période postopératoire en raison d'une réduction de la sécrétion endogène d'insuline et de son utilisation suite à la réponse de stress liée à la chirurgie. ${ }^{8}$ En outre, les besoins insuliniques nécessaires à supprimer la cétose/l'acidose métabolique pourraient être sous-estimés en raison des concentrations glycémiques proches de la normale associées à l'utilisation de SGLT$2 \mathrm{i}^{8}$

Le débat continue toutefois de faire rage quant à savoir si les SGLT-2i sont véritablement la cause de l'acidocétose diabétique dans un contexte de diabète de type $2 .{ }^{12}$ Dans une méta-analyse par Tang et coll., sur un total de dix études randomisées contrôlées éligibles englobant 13134 patients, seuls 14 événements d'acidocétose diabétique chez des patients atteints de diabète de type 2 et associés à l'utilisation de SGLT-2i ont été identifiés. Globalement, les taux d'événements étaient de $0,1 \%$ dans le groupe des utilisateurs de SGLT-2i, vs 0,06 \% dans les autres groupes de réduction de la glycémie (rapport de cotes $[\mathrm{RC}], 1,71$; intervalle de confiance [IC] $95 \%, 0,56$ à 5,2), et les analyses de sous-groupe ont démontré que les SGLT-2i n'étaient pas associés de manière significative à un risque accru d'acidocétose diabétique comparativement à un placebo (RC, 1,98; IC $95 \%, 0,56$ à 6,9). ${ }^{7}$ Une métaanalyse de Monami et coll. a inclus des études randomisées avec une durée de traitement $>12$ semaines chez des patients atteints de diabète de type 2 et comparant un SGLT-2i à un placebo ou à des comparateurs actifs. Sur les 72 études examinées, 9 ont rapporté au moins un événement d'acidocétose, avec 10157 et 5396 sujets dans les groupes SGLT-2i vs comparateurs, respectivement. Le nombre d'événements d'acidocétose diabétique était de 16 dans les groupes SGLT-2i et 6 dans les groupes comparateurs. Aucun signe de risque accru d'acidocétose n'a été observé pour les SGLT-2i en tant que classe de médicaments (RC, 1,14; IC $95 \%, 0,45$ à 2,$9 ; P=$ $0,78)$. Les auteurs concluent que le risque d'acidocétose diabétique associée aux SGLT-2i est négligeable si ces agents sont prescrits de manière adaptée aux patients atteints de diabète de type $2 .{ }^{13}$ Fadini a analysé et passé en revue 2397 présentations de cas d'acidocétose diabétique associés à l'utilisation de SGLT-2i entre 2014 et 2016 incluses dans le Système de communication des événements néfastes (Adverse Event Reporting System) de la FDA (Federal Drug Agency) américaine. Selon ses analyses, la divulgation accrue des cas d'acidocétose diabétique, associée à l'utilisation de SGLT-2i, était probablement davantage attribuable à l'augmentation de la pharmacovigilance et à un biais de communication plutôt qu'indicatrice de la véritable incidence, laquelle serait 
vraisemblablement déduite de façon plus juste à partir d'études concrètes et d'études cliniques d'envergure. ${ }^{14}$

Alors qu'il semble raisonnable de proposer des recommandations pour l'utilisation périopératoire des SGLT-2i en se fondant sur leur pharmacocinétique, comme le propose Lau et coll., l'absence de recommandations consensuelles provenant d'associations majeures de diabète, de chirurgie ou d'anesthésie pour l'utilisation périopératoire des SGLT-2i est notable, ce que nous avons récemment souligné ailleurs. ${ }^{15}$ Bien que l'American Association of Clinical Endocrinologists et l'American College of Endocrinology recommandent d'interrompre les SGLT-2i $24 \mathrm{~h}$ avant l'opération, ${ }^{16}$ la série de cas de Lau et coll. (et d'autres) ${ }^{8,12}$ soulignent que l'acidocétose diabétique euglycémique peut tout de même être précipitée malgré une interruption périopératoire de 48 $\mathrm{h}$ des SGLT-2i. ${ }^{12}$ Ceci met en exergue des lacunes importantes dans les connaissances cliniques entourant l'utilisation des SGLT-2i en période périopératoire et représente une occasion de réaliser des recherches d'amélioration de la qualité.

Conflicts of interest Sharon C. Peacock has no conflicts. Julie A. Lovshin has received consulting fees or speaking honorarium or both from Novo Nordisk, Eli Lilly \& Co, Merck Sharp \& Dohme, and AstraZeneca and grant support from Sanofi and Merck.

Editorial responsibility This submission was handled by Dr. Hilary P. Grocott, Editor-in-Chief, Canadian Journal of Anesthesia.

Funding There was no funding involved.

Conflit d'intérêt Sharon C. Peacock n'a aucun conflit à déclarer. Julie A. Lovshin a reçu des commissions de consultation, des honoraires de conférencière ou les deux de Novo Nordisk, Eli Lilly \& Co, Merck Sharp \& Dohme, et AstraZeneca, ainsi qu'une subvention d'aide de Sanofi et Merck.

Responsabilité éditoriale Cet article a été traité par Dr Hilary P. Grocott, rédacteur en chef, Journal canadien d'anesthésie.

Financement Aucun financement.

\section{References}

1. Committee Canadian Diabetes Association Clinical Practice Guidelines Expert. Pharmacologic management of type 2 diabetes: 2016 interim update. Can J Diabetes 2016; 40: 484-6.
2. Heerspink HJ, Perkins BA, Fitchett DH, Husain M, Cherney DZ. Sodium glucose cotransporter 2 inhibitors in the treatment of diabetes mellitus: cardiovascular and kidney effects, potential mechanisms, and clinical applications. Circulation 2016; 134: 752-72.

3. Ridderstråle $M$, Andersen KR, Zeller C, Kim G, Woerle HJ, Broedl UC; EMPA-REG H2H-SU trial investigators. Comparison of empagliflozin and glimepiride as add-on to metformin in patients with type 2 diabetes: a 104-week randomised, activecontrolled, double-blind, phase 3 trial. Lancet Diabetes Endocrinol 2015; 39: 691-700.

4. Zinman B, Wanner C, Lachin JM, et al. Empagliflozin, cardiovascular outcomes, and mortality in type 2 diabetes. $\mathrm{N}$ Engl J Med 2015; 373: 2117-28.

5. Wanner C, Inzucchi SE, Lachin JM, et al. Empagliozin and progression of kidney disease in type 2 diabetes. N Engl J Med 2016; 375: 323-34.

6. Neal B, Perkovic V, Mahaffey $K W$, et al. Canagliflozin and cardiovascular and renal events in type 2 diabetes. N Engl J Med 2017; 377: 644-57.

7. Tang H, Li D, Wang T, Zhai S, Song Y. Effect of sodium-glucose cotransporter 2 inhibitors on diabetic ketoacidosis among patients with type 2 diabetes: a meta-analysis of randomized controlled trials. Diabetes Care 2016; 39: e123-4.

8. Burke KR, Schumacher CA, Harpe SE. SGLT2 inhibitors: a systematic review of diabetic ketoacidosis and related risk factors in the primary literature. Pharmacotherapy 2017; 37: 187-94.

9. Lau A, Bruce S, Wang E, Ree R, Rondi K, Chau A. Perioperative implications of sodium-glucose cotransporter-2 inhibitors: a case series of euglycemic diabetic ketoacidosis in three patients after cardiac surgery. Can J Anesth 2018; 65: this issue. DOI: https:// doi.org/10.1007/s12630-017-1018-6.

10. Campbell JE, Drucker DJ. Islet $\alpha$ cells and glucagon-critical regulators of energy homeostasis. Nat Rev Endocrinol 2015; 11: 329-38.

11. Ferrannini E, Mark M, Mayoux E. CV protection in the EMPAREG OUTCOME trial: a "thrifty substrate" hypothesis. Diabetes Care 2016; 39: 1108-14.

12. Goldenberg RM, Berard LD, Cheng AY, et al. SGLT2 inhibitorassociated diabetic ketoacidosis: clinical review and recommendations for prevention and diagnosis. Clin Ther 2016; 38: 2654-64.e1.

13. Monami M, Nreu B, Zannoni S, Lualdi C, Mannucci E. Effects of SGLT-2 inhibitors on diabetic ketoacidosis: a meta-analysis of randomised controlled trials. Diabetes Res Clin Pract 2017; 130: 53-60.

14. Fadini GP, Bonora BM, Avogaro A. SGLT2 inhibitors and diabetic ketoacidosis: data from the FDA Adverse Event Reporting System. Diabetologia 2017; 60: 1385-9.

15. Peacock SC, Lovshin JA, Cherney DZ. Perioperative considerations for the use of sodium-glucose cotransporter-2 inhibitors in patients with type 2 diabetes. Anesth Analg 2017.

16. Handelsman $Y$, Henry RR, Bloomgarden ZT, et al. American Association of Clinical Endocrinologists and American College of Endocrinology position statement on the association of SGLT2 inhibitors and diabetic ketoacidosis. Endocr Pract 2016; 22: 753-62. 